Präv Gesundheitsf 2022 · 17:529-536 https://doi.org/10.1007/s11553-021-00906-0 Eingegangen: 14. Juni 2021

Angenommen: 20. September 2021

Online publiziert: 13 . Oktober 2021

(c) Der/die Autor(en) 2021

\author{
Gerhard Müller ${ }^{1}(\mathbb{D}) \cdot$ Felix Kunst ${ }^{2} \cdot$ Norman Stutzig $^{2}$. \\ Monika Heinzel-Gutenbrenner ${ }^{5}$ Manuela Bombana ${ }^{1,6} \cdot$ Marco Giurgiu $^{3,4}$. \\ Klaus Bös ${ }^{3}$. Darko Jekauc ${ }^{3}$ \\ 'Geschäftsbereich Vorsorge \& Prävention, AOK Baden-Württemberg, Stuttgart, Deutschland \\ ${ }^{2}$ Institut für Sport- und Bewegungswissenschaft, Universität Stuttgart, Stuttgart, Deutschland \\ ${ }^{3}$ Institut für Sport und Sportwissenschaft, Karlsruher Institut für Technologie, Karlsruhe, Deutschland \\ ${ }^{4}$ Institut für Psychiatrische und Psychosomatische Psychotherapie, Zentralinstitut für Seelische \\ Gesundheit, Universität Heidelberg, Mannheim, Deutschland \\ ${ }^{5}$ Dr. Monika Heinzel-Gutenbrunner, MH Statistikberatung, Marburg, Deutschland \\ ${ }^{6}$ Abteilung Allgemeinmedizin und Versorgungsforschung, Universitätsklinikum Heidelberg, Heidelberg, \\ Deutschland
}

\section{Der Unterschied zwischen Sagen und Tun beim Trainingsfleiß}

\section{Effekte von Motiven sportlicher Aktivität, Alter, Geschlecht und Schmerzen auf den Trainingsumfang sportlicher Aktivitäten}

\section{Einleitung}

Warum sind Menschen mehr oder weniger sportlich aktiv? Diese Frage ist von zentraler Bedeutung, denn Bewegungsmangel ist einer der bedeutendsten Risikofaktoren in Deutschland und weltweit für die Entwicklung von chronischen Erkrankungen und trägt zu einer erheblichen Reduzierung der Lebenserwartung bei. Er steht an vierter Stelle der Risikofaktoren für eine erhöhte Sterblichkeit $(6 \%$ aller weltweiten Todesfälle, 7,7\% in Ländern mit hohem Einkommen [30]). So wurden 2015 in Deutschland $10 \%$ der durch koronare Herzkrankheit verlorenen Lebensjahre, $17 \%$ der durch Diabetes mellitus verlorenen Jahre, $15 \%$ der durch Darmkrebs verlorenen Jahre und $10 \%$ der durch Brustkrebs verlorenen Jahre durch Bewegungsmangel verursacht [7]. Diese und andere chronische Krankheiten stehen aber auch für rund 70-80\% aller Krankheitskosten und gelten nach aktuellem Forschungsstand als nicht heilbar - folgerichtig setzt die WHO(World Health Organisation)-Europa auf Prävention, wenn es um die Dämpfung der Krankheitskosten in den kommenden Jahren geht [9, 29]. Das Problem scheint sich zudem in den nächsten Jahren eher zu verschärfen als rückläufig zu sein: So wird mit einem Anstieg der Krankheitskosten in den EU28-Staaten von rund $50 \%$ im Zeitraum von 2015 bis 2030 aufgrund von Bewegungsmangel ausgegangen [12].

Die Frage, warum Menschen sportlich aktiv sind, scheint vordergründig beantwortet zu sein [7]. Das Gesundheitsmotiv wird in Befragungen überwiegend als stärkstes/häufigstes Motiv von den Befragten genannt $[5,14,20]$. Danach folgen Motive wie Freude/Spaß haben, Figur/Aussehen, Erholung u. a. Der gesellschaftliche Kontakt und auch das Wettkampfmotiv werden dagegen seltener als Motiv für sportliche Aktivitäten genannt [1]. Diese Ergebnisse haben zu einer primär gesundheitsorientierten Ausrichtung von Sportangeboten geführt und damit andere Motive in den Hintergrund gedrängt [14]. Die Fokussierung auf das Gesundheitsmotiv ist aber auf Basis von Befragungsergebnissen zu den Motiven sportlicher Aktivitäten letztlich nicht begründbar, denn nur weil ein Motiv in einer Befragung als wichtig für sportliche Aktivitäten benannt wird („das Motiv ist mir ganz wichtig“), muss es nicht zwingend auch zu einem höheren Trainingsumfang führen („deshalb trai- niere ich auch mehr"). Der Unterschied zwischen Sagen (das Motiv ist mir ganz wichtig) und Tun (deshalb trainiere ich auch mehr) wird je nach Motiv und Subgruppe (Alter, Geschlecht u.a.) vermutlich mehr oder weniger groß ausfallen $[11,14,23,25]$.

Befragungsergebnisse zu den Motiven sportlicher Aktivitäten allein greifen hier zu kurz, wenn wir die Motive mit den stärksten Effekten auf den Trainingsumfang ermitteln wollen.

Nur für die intrinsisch gelagerten Motive Freude, Spaß, Kompetenzerleben an sportlichen Aktivitäten ist die Forschungslage relativ eindeutig: Werden sportliche Aktivitäten um ihrer selbst willen ausgeführt, wirkt sich dies positiv auf die Trainingshäufigkeit, -adhärenz aus [25].

Die Ergebnisse bestätigen damit tendenziell die Selbstbestimmungstheorie („self-determination theory“, SDT) von Ryan und Deci, dass sich der Grad der Selbstbestimmung (intrinsische Motivation $=$ maximale Selbstbestimmung) positiv auf die Bereitschaft zu einer Handlungsausführung (Adhärenz sportlicher Aktivitäten) auswirkt [22]. Diese Ergebnisse stehen aber auch in Übereinstimmung mit der Bedeutung des 


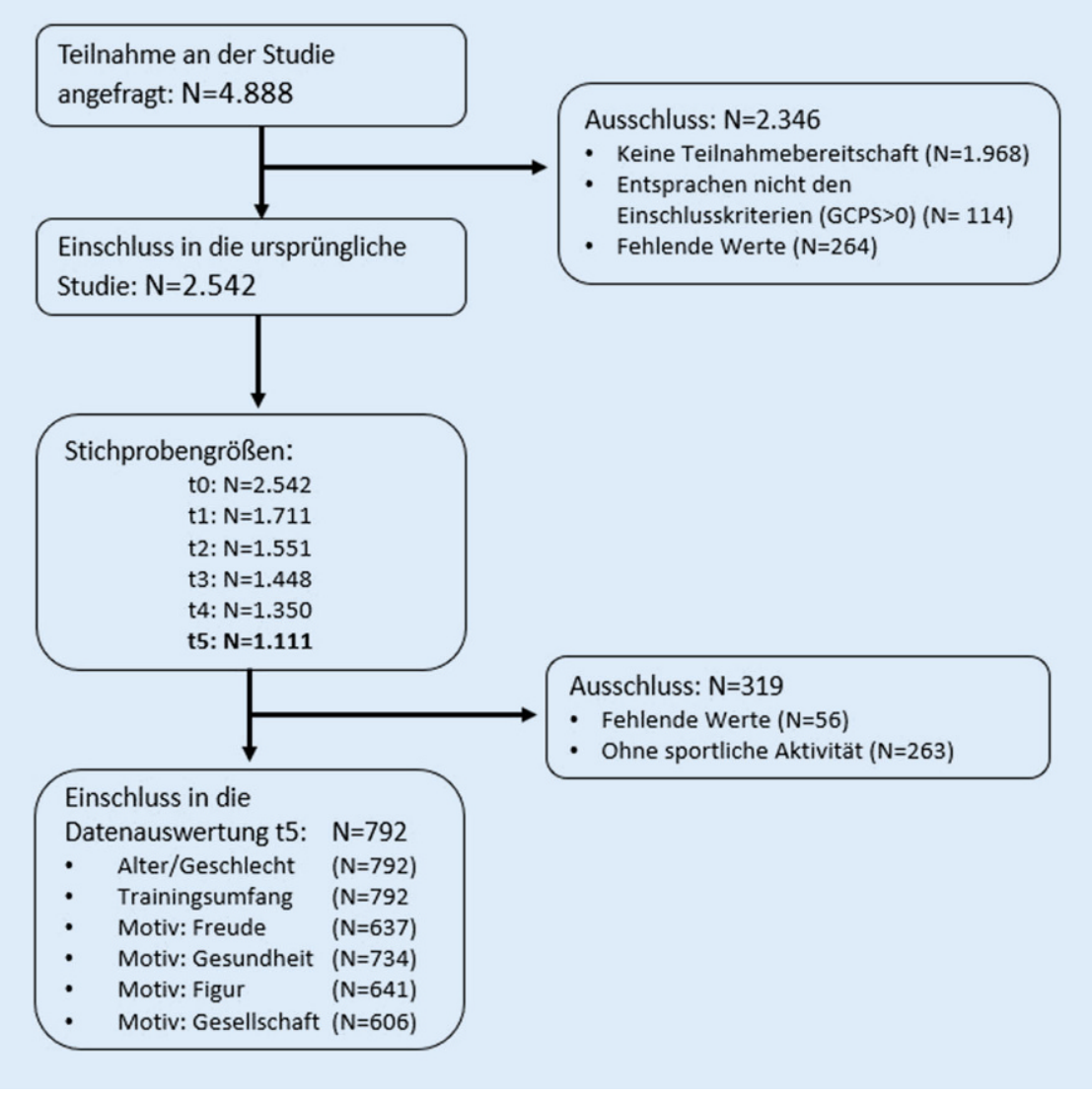

Abb. 1 \ Stichprobengrößen zu den Messzeitpunkten

zeitlichen Horizonts (Diskontierung der Belohnung, „delay discounting “) für die Stärke einer Motivation. So erfolgt die Belohnung (Freude empfinden) beim Motiv Freude direkt durch das positive Erleben bei der sportlichen Aktivität ohne zeitliche Verzögerung und nicht erst als Ergebnis des Trainings zu einem späteren Zeitpunkt (z.B. Gesundheit) und damit diskontiert, abgeschwächt $[16,23]$.

Über das Motiv Freude hinaus ist der Forschungsstand zum Effekt von Motiven auf den Trainingsumfang sportlicher Aktivitäten für unterschiedliche Subgruppen (Alter, Geschlecht u. a.) unklar und auch die Selbstbestimmungstheorie liefert hier keine konsistenten Erkenntnisse [11, 14, 23, 25].

Soll der Trainingsumfang sportlicher Aktivitäten gesteigert und damit Bewegungsmangel reduziert werden, braucht die Trainingspraxis Antworten auf die Frage der Treiber sportlicher Aktivitäten. Soll der nächste Gesundheitskurs neben Spaß den Gesellschafts-, den Ge- sundheitsgedanken oder eher die Verbesserung der Figur in den Vordergrund stellen, wenn ein möglichst hohes Trainingsvolumen, eine hohe Adhärenz das Ziel sind? Stellen Schmerzen eher einen Motivator oder eine Barriere für sportliche Aktivitäten dar [4, 19, 24]? Und unterscheiden sich Männer und Frauen unterschiedlichen Alters in diesen Fragen? Diese Fragen sind zu beantworten, wenn wir effektiv Bewegungsmangel reduzieren wollen.

In dieser Untersuchung wurden deshalb die Effekte der Motive Gesundheit, Figur, Freude und Gesellschaft auf den Trainingsumfang sportlich aktiver Männer und Frauen unterschiedlichen Alters mit unterschiedlicher Rückenschmerzproblematik untersucht.

\section{Studiendesign und Unter- suchungsmethoden}

Die Daten stammen aus einer Multicenterstudie (39 Standorte in BadenWürttemberg) zur Prüfung der gesund- heitlichen und ökonomischen Effekte eines Gesundheitskurses (Rückentraining) einer gesetzlichen Krankenversicherung [17]. Der bei der Ethikkommission der Universität Greifswald für diese Untersuchung eingereichte Ethikantrag erhielt am 03.06.2008 ein uneingeschränktes positives Votum (ID 33/08). Sechs Jahre nach Beendigung (2014/2015) der Intervention haben wir die Untersuchungsteilnehmer erstmals $\mathrm{zu}$ den Motiven ihrer sportlichen Aktivitäten und ihrem Trainingsumfang sportlicher Aktivitäten befragt. Voraussetzung für die Teilnahme an der Studie war, dass die Teilnehmer/innen im letzten halben Jahr sportlich aktiv waren.

\section{Stichprobe}

Von Oktober 2007 bis März 2008 wurden insgesamt 4888 Versicherte einer gesetzlichen Krankenversicherung zu ihrer Teilnahmebereitschaft an der Untersuchung schriftlich befragt. 2542 (52\%) von ihnen konnten in die ursprüngliche Untersuchung einbezogen werden. Dieser Untersuchung liegen die Daten der letzten von sechs Befragungen zugrunde ( $\mathrm{t} 5)$, die 6 Jahre nach der Intervention durchgeführt wurde. In dieser letzten Befragung wurde erstmals auch nach den Motiven sportlicher Aktivität gefragt, neben Fragen zu sportlichen Aktivitäten und der Stärke der Rückenschmerzen im letzten halben Jahr. Insgesamt konnten die Daten von 792 Untersuchungsteilnehmern/-innen in die Auswertung einbezogen werden $(n=1111 \mathrm{zu} \mathrm{t} 5$, Ausschluss: $n=56$ aufgrund fehlender Werte/keine Angaben zu Trainingsumfang oder Motiven, ohne sportliche Aktivität $n=263$, s. $\bullet$ Abb. 1).

\section{Messinstrumente}

Das Alter, Geschlecht, Berufstätigkeit und Schulabschluss (liegt nur eingeschränkt vor) wurden über die Routinedaten des Krankenversicherers selektiert. Der Trainingsumfang der sportlichen Aktivitäten, die Ausprägung der Motive und Rückenschmerzen wurden über Befragung erhoben.

Auf der Basis der Ergebnisse bisheriger Befragungen wurden die Ausprä- 
Präv Gesundheitsf 2022 · 17:529-536 https://doi.org/10.1007/s11553-021-00906-0

(c) Der/die Autor(en) 2021

G. Müller · F. Kunst · N. Stutzig · M. Heinzel-Gutenbrenner · M. Bombana · M. Giurgiu · K. Bös · D. Jekauc

\section{Der Unterschied zwischen Sagen und Tun beim Trainingsfleiß. Effekte von Motiven sportlicher Aktivität, Alter, Geschlecht und Schmerzen auf den Trainingsumfang sportlicher Aktivitäten}

\section{Zusammenfassung}

Hintergrund. Gesundheit, Freude/Spaß, Figur und Gesellschaft sind Motive für sportliche Aktivitäten. Es ist unklar, welcher Zusammenhang zwischen ihrer Ausprägung, Alter, Geschlecht, Schmerzen und Trainingsumfang besteht.

Material und Methoden. In dieser Untersuchung wurden 792 sportlich aktive Männer und Frauen $(68 \%$ Frauen und $32 \%$ Männer, Alter $M=53,5$ Jahren, $S D=11,9$, Range 24-85 Jahre) zur Ausprägung ihrer Motive für sportliche Aktivitäten, ihren Rückenschmerzen und dem Trainingsumfang ihrer sportlichen Aktivitäten befragt. Zur Überprüfung der Effekte auf den Trainingsumfang wurden Regressionsanalysen gerechnet.
Ergebnisse. Das Motiv Gesundheit war bei Männern und Frauen das stärkste Motiv für ihre sportlichen Aktivitäten, ohne einen signifikanten Effekt auf den Trainingsumfang zu haben. Bei Frauen hatten die Motive Freude ( $B_{\text {Freude }}=0,23, t=3,6, p<0,001$, $r=0,25)$, Figur ( $B_{\text {Figur }}=0,127, t=2,0, p=0,05$, $r=0,16)$ und Alter ( $B_{\text {Alter }}=0,03, t=2,5$, $p=0,01, r=0,17$ ) einen kleinen (Figur, Alter) bis mittleren Effekt (Freude) auf den Trainingsumfang. Bei Männern hatten nur Rückenschmerzen einen signifikanten positiven Effekt ( $B_{\text {Rückenschmerzen }}=1,4, t=4,2$, $p<0,001, r=0,35)$ auf den Trainingsumfang (starker Effekt).

Diskussion und Schlussfolgerung. Bei sportlich aktiven Frauen fördert die beim
Training empfundene Freude (intrinsische Motivation) und der Wunsch nach einer besseren Figur (introjizierte Regulation = externen Anforderungen entsprechen) den Trainingsumfang. Für sportlich aktive Männer steht möglicherweise die Lösung des gesundheitlichen Problems im Vordergrund: Je größer das Problem (Rückenschmerzen) desto höher ist der Trainingsumfang. Vermutlich haben sie die Erfahrung gemacht, dass körperliches Training die Rückenschmerzen reduziert.

Schlüsselwörter

Motive sportlicher Aktivitäten · Trainingsumfang · Rückenschmerzen - Trainingspraxis . Gesundheitskurse

\section{The difference between saying and doing when it comes to training volume. Effect of motives of sport activity, age, gender and pain on training volume}

\section{Abstract}

Background. Health, joy/fun, body shape and society are motives for physical exercise. So far, the impact of the strength of these factors on the training scope is unclear. The study aims to analyse the association between these motives, age, gender, back pain and training volume.

Materials and methods. In this study, 792 participants $(68 \%$ women and $32 \%$ men, mean age 53.5 years, standard deviation 11.9, range $24-85$ years) were asked about their motives for physical activities, their back pain and their volume of training. Regression analyses were performed to assess the effects of the motives on training volume. Results. In women, the motives joy $\left(B_{\text {joy }}=0.23, t=3.6, p<0.001, r=0.25\right)$, body shape $\left(\mathrm{B}_{\text {body shape }}=0.127, \mathrm{t}=2.0, p=0.05\right.$, $r=0.16)$ and age $\left(B_{\text {age }}=0.03, t=2.5, p=0.01\right.$, $r=0.17$ ) had small (age, body shape) to medium effects (joy) on training volume. In men, only back pain had a significant positive effect $\left(B_{\text {back pain }}=1.4, t=4.2, p<0.001\right.$, $r=0.35$ ) on training volume (strong effect). Discussion and conclusion. For active women, the pleasure felt during training (intrinsic motivation) and the desire for a better figure (introjected regulation $=$ meeting external requirements) promote the amount of training. For active men, the solution to the health problem is possibly in the foreground: the greater the problem (back pain), the higher the training volume. Presumably, they have made the experience that physical training reduces back pain.

Keywords

Motives of physical exercise - Amount of training $\cdot$ Back pain $\cdot$ Training practice $\cdot$ Health courses gung der gängigsten Motive im Bereich des Gesundheitssports (Gesundheit, Figur, Gesellschaft, Freude) auf einer 11erSkala $(0=$ trifft nicht $\mathrm{zu}$ bis $\mathrm{zu} 10=$ trifft völlig zu) über die Frage „Warum sind Sie sportlich aktiv?" (Bitte kreuzen Sie an, wie sehr die Aussagen auf Sie zutreffen: „Ich will etwas für meine Gesundheit tun“; „Mir macht Sport Freude“; „Ich will etwas für meine Figur tun“; „Mir ist Gesellschaft beim Sport wichtig") erhoben $[3,5,20]$.

Der Trainingsumfang im vergangenen halben Jahr wurde für unterschiedliche
Kategorien sportlicher Aktivität erfragt (Ausdauer, Fitness, Rückentraining, Ballsport, Entspannung, Gymnastik). Wenn sportliche Aktivitäten unter „sonstige sportliche Aktivitäten“ benannt wurden, erfolgte eine Zuordnung durch zwei Sportwissenschaftlern zu den passenden Kategorien oder zur Kategorie „sonstige sportliche Aktivitäten“ [18].

Rückenschmerzen wurden über den „chronic pain grade questionnaire“ (CPGQ) erfragt [13]. Auf Basis der aktuellen, durchschnittlichen und stärksten Rückenschmerzen im letzten halben Jahr, der Anzahl an Tagen mit Beschwerden aufgrund von Rückenschmerzen und der funktionellen Einschränkungen aufgrund von Rückenschmerzen in Alltag, Beruf und Freizeit werden 5 Grade der Rückenbeschwerden unterschieden: Grad 0 (keine Rückenschmerzen), Grad 1 (leichte Rückenschmerzen), Grad 2 (starke Rückenschmerzen), Grad 3 (mittlere Funktionseinschränkungen), Grad 4 (starke Funktionseinschränkungen [13]). 
Tab. 1 Soziodemographische Beschreibung der Stichprobe

\begin{tabular}{|c|c|c|c|c|c|}
\hline & & Män & $n=254)$ & Frau & $=538)$ \\
\hline & & $n$ & $\%$ & $n$ & $\%$ \\
\hline Altersgruppie- & 20-35 Jahre & 10 & 3,9 & 53 & 9,9 \\
\hline rungen & 36-50 Jahre & 65 & 25,6 & 166 & 30,9 \\
\hline & 51-65 Jahre & 116 & 45,7 & 247 & 45,9 \\
\hline & 66-85 Jahre & 63 & 24,8 & 72 & 13,4 \\
\hline Schul- & Ohne Schulabschluss & 1 & 1,3 & 0 & 0,0 \\
\hline abschluss ${ }^{\mathrm{a}}$ & Haupt-/Volksschulabschluss & 48 & 60,0 & 67 & 30,7 \\
\hline & Mittlere Reife & 24 & 30,0 & 101 & 46,3 \\
\hline & Abitur/Fachabitur & 7 & 8,8 & 50 & 22,9 \\
\hline Beschäfti- & Nicht berufstätig & 57 & 22,4 & 157 & 29,2 \\
\hline gungsstatus & Berufstätig & 197 & 77,6 & 381 & 70,8 \\
\hline
\end{tabular}

Tab. 2 Sportliche Aktivität (Trainingsumfang in Stunden pro Woche)

\begin{tabular}{lllll|l|l|l}
\hline & Frauen & \multicolumn{7}{c}{ Männer } \\
\hline Sportliche Aktivität & $\boldsymbol{M}$ & SD & $\%$ & $\boldsymbol{M}$ & SD & $\%$ \\
\hline Ausdauertraining & 2,27 & 2,6 & 63 & 3,36 & 3,87 & 71 \\
Fitnesstraining & 0,55 & 1 & 15 & 0,43 & 0,87 & 9 \\
\hline Rückentraining & 0,38 & 0,8 & 10 & 0,58 & 1,16 & 12 \\
Gymnastiktraining & 0,13 & 0,4 & 4 & 0,05 & 0,25 & 1 \\
\hline Ballsportarten Training & 0,07 & 0,4 & 2 & 0,18 & 0,67 & 4 \\
Entspannungstraining & 0,11 & 0,4 & 3 & 0,00 & 0,03 & 0 \\
\hline Sonstiges Training & 0,10 & 0,5 & 3 & 0,14 & 0,86 & 3 \\
\hline Trainingsumfang insgesamt & 3,60 & 3,1 & 100 & 4,7 & 4,3 & 100
\end{tabular}

Tab. 3 Ausprägung der Rückenschmerzen

\begin{tabular}{|llll} 
& & Männer & Frauen \\
\hline GCPS & Keine Rückenschmerzen & \% & $\%$ \\
\hline & Niedrige Schmerzintensität & 3,9 & 4,6 \\
\hline & Hohe Schmerzintensität & 62,2 & 63,2 \\
\hline & Mäßige Funktionseinschränkung & 16,5 & 16,7 \\
& Schwere Funktionseinschränkung & 13,4 & 11,3 \\
\hline GCPS "graded chronic pain status" & 3,9 & 4,1 \\
\hline
\end{tabular}

\section{Statistische Analyse}

Als deskriptive Daten wurden Mittelwerte und Standardabweichungen berechnet. Geschlechterunterschiede in den Motivausprägungen wurden über t-Tests für unabhängige Stichproben geprüft. Zur Analyse der Effektstärken wurden Cohen's d berechnet [2]. Zur Überprüfung des Zusammenhangs von Motivstärke, Alter, Rückenschmerz (unabhängige Variablen) und Trainingsumfang (abhängige Variable) wurden Regressionsanalysen für Männer und Frauen getrennt gerechnet: (Trainingsum-

\section{Ergebnisse}

\section{Beschreibung der Stichprobe}

Soziodemographie. Die Stichprobe bestand aus 792 Untersuchungsteilnehmern/innen (Alter $M=53,5$ Jahren, $S D=11,9$, Range 24-85 Jahre), $68 \%$ Frauen (Alter $\mathrm{M}=52,1$ Jahre, $\mathrm{SD}=11,7$ ) und $32 \%$ Männer (Alter $M=56,5$ Jahre, $\mathrm{SD}=11,7) .22,9 \%$ der Frauen hatten Abitur/Fachabitur (Männer 8,8\%), 46,3\% Mittlere Reife (Männer 30\%), 30,7\% Haupt-/Volksschulabschluss (Männer $60 \%)$ und keine Frau war ohne Schulabschluss (Männer 1,3\%). $71 \%$ der Frauen waren beschäftigt (Männer $78 \%$; - Tab. 1).

Sportliche Aktivität. Frauen trainierten durchschnittlich 3,6h $(S D=3,2)$ pro Woche, Männer $4,7 \mathrm{~h}(S D=4,3)$, $(t(790)=4,23, p<0,001, d=0,323)$. Männer waren in Ausdauertraining, Rückentraining und Ballsportarten aktiver, während Frauen eher Fitnesstraining, Gymnastik und Entspannungstraining absolvierten. Ausdauertraining hatte bei Frauen und Männern den größten Anteil am Trainingsumfang (• Tab. 2).

Motive des Sporttreibens. Das Gesundheitsmotiv war das stärkste Motiv $(M=8,57, S D=1,95)$, gefolgt von den Motiven Figur $(M=7,37, S D=2,70)$, Freude $(M=7,13, S D=2,68)$ und Gesellschaft $(M=4,90, S D=3,45)$. Bei Frauen waren alle Motive mit Ausnahme des Motivs Freude stärker ausgeprägt als bei den Männern, der Effekt des Geschlechts war klein (s. - Abb. 2): Motiv Freude $t(635)=-1,34, p=0,182, d=-0,112$; Motiv Gesundheit $t(732)=4,22, p<0,001$, $d=0,362$; Motiv Figur $t(639)=4,64$; $p<0,001, d=0,398$; Motiv Gesellschaft $t(604)=2,64, p=0,022, d=0,199$.

Rückenschmerzen. Männer $(M=1,51$, $S D=0,91)$ und Frauen $(M=1,47, S D$ $0,90)$ unterschieden sich nicht in der Ausprägung der Rückenschmerzen $(t(790)=-0,602, p=0,547)$, • Tab. 3 . 


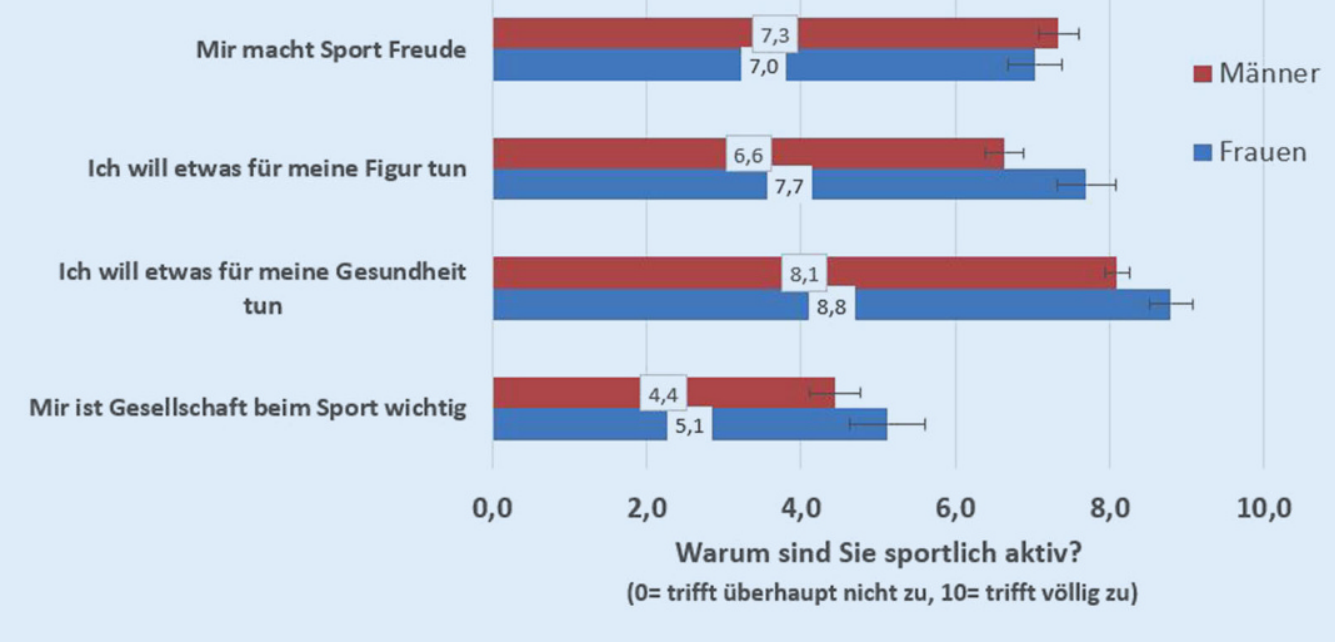

Abb. $2 \triangleleft$ Ausprägung der Motive für sportliche Aktivitäten bei Männern und Frauen

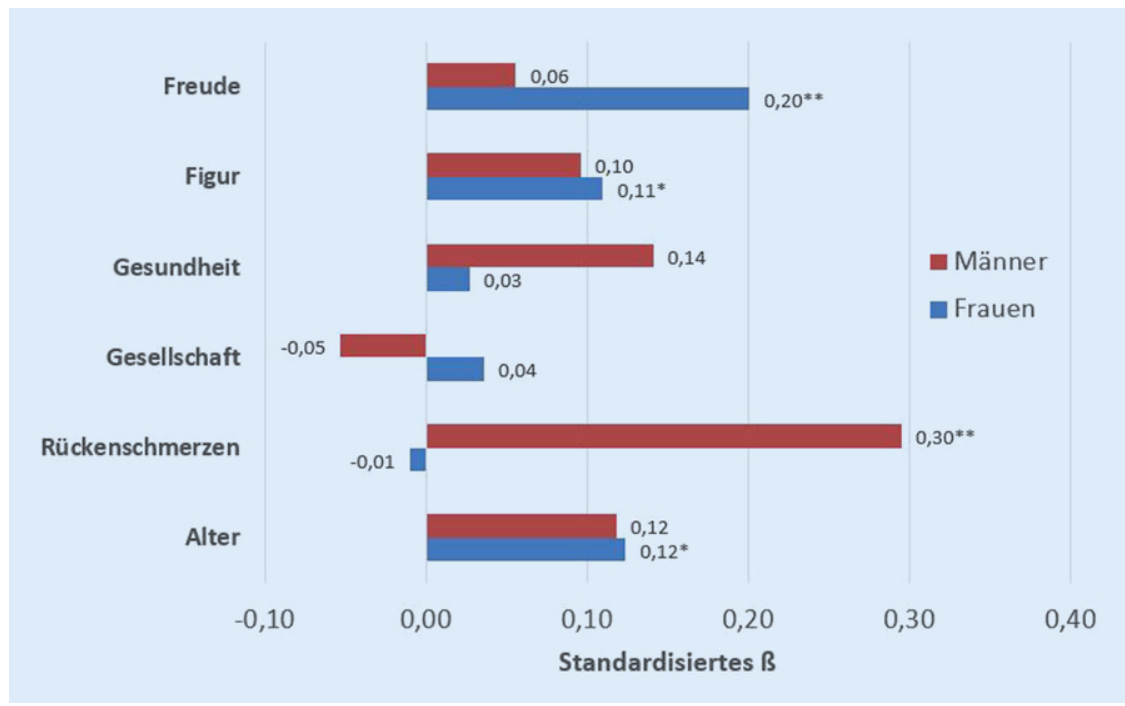

Abb. $3 \Delta$ Standardisierte $\beta$-Werte der unabhängigen Variablen Alter, Rückenschmerzen und der Motive sportlicher Aktivitäten (Freude, Figur, Gesundheit, Gesellschaft) auf die abhängige Variable Trainingsumfang $\left({ }^{*} p \leq 0,05,{ }^{* *} p<0,001\right)$ bei Männern und Frauen

\section{Effekte auf den Trainingsumfang}

Frauen. Bei Frauen hatten die Motive Freude $\left(\mathrm{B}_{\text {Freude }}=0,23, t=3,6, p<0,001\right.$, $\mathrm{r}=0,25)$ und Figur $\left(\mathrm{B}_{\text {Figur }}=0,127, t=2,0\right.$, $p=0,05, \mathrm{r}=0,16)$ und das Alter $\left(\mathrm{B}_{\text {Alter }}=\right.$ $0,032, t=2,5, p=0,012, \mathrm{r}=0,17)$ einen signifikanten Effekt auf den Trainingsumfang (•Tab. 4; • Abb. 3). Die Regressionsanalyse zeigt die Zusammenhänge zwischen den unabhängigen Variablen und dem Trainingsumfang auf. So steigt mit jedem Skalenpunkt beim Motiv Freude der Trainingsumfang um 13,5 min $\left(B_{\text {Freude }}{ }^{\star} 60 \mathrm{~min}\right)$, beim Motiv Figur um $7,6 \mathrm{~min}\left(\mathrm{~B}_{\text {Figur }}{ }^{*} 60 \mathrm{~min}\right)$ pro Woche und mit jeder Zunahme des Alters um ein Jahr erhöhte sich der Trainingsumfang um 1,9 min.

Das Modell bei den Frauen hat mit einem $R^{2}=0,10$ (korrigiertes $R^{2}=0,09$ ) eine geringe bis mittlere Anpassungsgüte.

Männer. Bei Männern hatten nur Rückenschmerzen einen signifikanten Effekt ( $B_{\text {Rückenschmerzen }}=1,4, \quad t=4,2, \quad p<0,001$, $\mathrm{r}=0,35)$ auf den Trainingsumfang (- Tab. 4; - Abb. 3). Die Regressionsanalyse zeigt, dass mit jedem Grad Rückenschmerzen der Trainingsumfang um $84 \mathrm{~min}$ (Bückenschmerzen $* 60 \mathrm{~min}$ ) pro Woche steigt. Das Modell hat mit einem $R^{2}=0,16$ (korrigiertes $R^{2}=0,13$ ) auch bei den Männern eine mittlere Anpassungsgüte.

\section{Diskussion}

Ziel der Untersuchung war es, den $\mathrm{Zu}$ sammenhang zwischen Motiven sportlicher Aktivitäten, Alter, Geschlecht, Rückenschmerzen und Trainingsumfang zu analysieren.

\section{Zentrale Ergebnisse}

Gesundheit war zwar das Motiv mit der stärksten Ausprägung in der Befragung, hatte aber keinen signifikanten Effekt auf den Trainingsumfang, weder bei Frauen noch bei Männern.

Bei den Frauen waren Freude, Figur und das Alter signifikante Prädiktoren für den Trainingsumfang. Freude hatte von allen Variablen bei den Frauen den stärksten Effekt auf den Trainingsumfang ( $r=0,25$, mittlerer Effekt). Bei den Männern erwiesen sich nur Rückenschmerzen als signifikanter Prädiktor für den Trainingsumfang $(r=0,35$, starker Effekt).

\section{Diskussion der zentralen Ergebnisse}

Die höchste Ausprägung bei der Befragung hatte, wie erwartet, das Motiv Gesundheit, gefolgt von den Motiven Figur, 
Tab. 4 Effekte von Alter, Rückenschmerzen und Motiven sportlicher Aktivitäten auf den Trainingsumfang bei Männern und Frauen

\begin{tabular}{|c|c|c|c|c|c|c|c|c|c|c|}
\hline & \multicolumn{5}{|l|}{ Frauen } & \multicolumn{5}{|c|}{ Männer } \\
\hline & B & SE & $\boldsymbol{\beta}$ & $\boldsymbol{t}$ & $\boldsymbol{P}$ & B & SE & $\beta$ & $\boldsymbol{t}$ & $p$ \\
\hline (Konstante) & $-1,165$ & 1,014 & - & $-1,150$ & 0,251 & $-3,491$ & 1,942 & - & $-1,798$ & 0,074 \\
\hline Alter & 0,032 & 0,013 & 0,124 & 2,516 & 0,012 & 0,044 & 0,026 & 0,118 & 1,690 & 0,093 \\
\hline Mir macht Sport Freude & 0,225 & 0,063 & 0,201 & 3,596 & $<0,001$ & 0,095 & 0,142 & 0,056 & 0,671 & 0,503 \\
\hline Ich will etwas für meine Gesundheit tun & 0,046 & 0,096 & 0,027 & 0,480 & 0,632 & 0,275 & 0,176 & 0,141 & 1,561 & 0,120 \\
\hline Ich will etwas für meine Figur tun & 0,127 & 0,065 & 0,110 & 1,967 & 0,050 & 0,153 & 0,137 & 0,096 & 1,119 & 0,265 \\
\hline Mir ist Gesellschaft beim Sport wichtig & 0,032 & 0,047 & 0,036 & 0,681 & 0,496 & $-0,067$ & 0,100 & $-0,053$ & $-0,665$ & 0,507 \\
\hline Rückenschmerzen & $-0,034$ & 0,165 & $-0,010$ & $-0,204$ & 0,838 & 1,399 & 0,330 & 0,295 & 4,246 & $<0,001$ \\
\hline
\end{tabular}

Freude und Gesellschaft. Frauen wiesen mit Ausnahme des Motivs Freude bei allen anderen Motiven signifikant höhere Werte auf als Männer. Diese Ergebnisse bestätigen die Ergebnisse vergleichbarer Studien [1, 3, 5, 14, 20].

Obwohl die Untersuchungsteilnehmer/-innen das Motiv Gesundheit als stärkstes Motiv für ihre körperlichen Aktivitäten benannten, hatte die Stärke des Motivs Gesundheit keinen signifikanten Effekt auf den Trainingsumfang. Dieses Ergebnis steht in guter Übereinstimmung mit dem aktuellen Forschungsstand [25]. Insgesamt zeigt die überwiegende Anzahl an Untersuchungen keinen Zusammenhang zwischen der Ausprägung des Gesundheitsmotivs und dem Trainingsumfang auf [25]. Der Grund für diese heterogenen Ergebnisse könnte in der unterschiedlichen Erfassung des Motivs Gesundheit liegen oder der starken Diskontierung des Motivs: Eine bessere Gesundheit ist das Ergebnis jahrelangen Trainings und bis zum Eintritt einer schweren Erkrankung weitgehend abstrakt [23, 25].

Der positive Effekt des Motivs Freude auf den Trainingsumfang bei den Frauen bestätigt den Forschungsstand, dass intrinsische Motivation die Persistenz im Verhalten stärker fördern als extrinsische Motivation [21, 23, 26]. Die große Nähe des Motivs Freude und der intrinsischen Motivation zeigte sich auch in der Untersuchung von Wienke und Jekauc [27], nach der Freude und intrinsische Motivation die beiden Seiten einer Medaille zu sein scheinen: Soziales Erleben und Kompetenzerleben als Grundbedürfnisse innerhalb der SDT waren Prädiktoren sowohl für Freude als auch für intrinsische Motivation. Die Ergebnisse bestätigen aber auch die Ergebnisse der Bad Schönborner Studie, dass Gesundheit eher ein Einsteiger- und Freude ein Dabeibleibermotiv ist [28].

Neben Freude zeigte das Motiv Figur bei den Frauen einen signifikanten, aber kleineren Effekt auf den Trainingsumfang. Bei Frauen scheint der Trainingsumfang stärker durch äußere Anforderungen (introjizierte Regulation), wie z. B. Aussehen, Figur, geprägt zu sein als bei Männer [10, 11]. Darüber hinaus erfolgt die Belohnung des Trainings (bessere Figur) bei diesem Motiv früher als beim Motiv Gesundheit. Der Effekt des Alters steht zumindest teilweise in Übereinstimmung mit dem Forschungsstand. So steigt der prozentuale Anteil der Frauen, die den Bewegungsempfehlungen der WHO entsprechen, von der Altersgruppe der 30- bis 44-jährigen Frauen von $22,3 \%$ auf $29 \%$ bei den über 65-jährigen Frauen in der oberen Bildungsgruppe (untere Bildungsgruppe von $11,1 \%$ auf $12,1 \%$, mittlere Bildungsgruppe von $15 \%$ auf $19 \%$ [7]).

Dass bei Männern nur die Stärke der Rückenschmerzen einen signifikanten positiven Effekt auf den Trainingsumfang hatte, erscheint erstaunlich. Wir wissen aber, dass es zwischen körperlichem Training und Rückgang der Rückenschmerzen ein Dosis-WirkungsZusammenhang besteht [6]. Die Wahrscheinlichkeit ist damit groß, dass sie überwiegend genau diese Erfahrung gemacht haben: Je mehr ich körperlich aktiv bin, desto besser werden meine Rückenschmerzen. Für sportlich aktive Männer steht damit möglicherweise die Lösung des gesundheitlichen Problems im Vordergrund. Schmerzen werden aber sowohl als Motivatoren als auch als Barrieren für sportliche Aktivitäten diskutiert $[19,24]$. Insofern erstaunt es umgekehrt auch nicht, dass bei Frauen genau der entgegengesetzte Effekt zu beobachten war: Die Höhe der Rückenschmerzen hatte tendenziell einen negativen Effekt (n.s.) auf den Trainingsumfang. Das standardisierte $\beta$ ist mit $-0,01 \quad(r=0,06)$ allerdings klein, so dass es fraglich ist, ob hier überhaupt ein Effekt vorliegt. Die Analyse der Zusammenhänge ist insbesondere bei den Männern unterpowert $(n=254)$ für die Detektion von kleineren Effekten (ein $n$ von 882 wäre ausreichend bei sechs Prädiktoren, $\left.\alpha=0,05, \beta=0,90, f^{2}=0,02\right)$. Zukünftige Studien sollten deshalb die Effekte an ausreichend großen Stichproben bei Männern und Frauen prüfen.

Die Stärke der Untersuchung liegt dennoch in der relativ großen Stichprobe und dem Erfassen von Motivstärke und Trainingsumfang. Die Ergebnisse stammen aber nicht aus einer Zufallsstichprobe und sind der Stichprobe entsprechend nur für sportlich aktive Erwachsene (eher typische Gesundheitskursteilnehmer/-innen) generalisierbar. Eine weitere Limitation ist, dass die Motivstärke nicht über einen standardisierten Fragebogen erfragt wurde, sondern die Motivstärke für jedes Motiv direkt erfragt wurde (One-Item). Die Ladungen der Items waren im Berner Motiv- und Zielinventar im Freizeit- und Gesundheitssport relativ hoch $(\geq 0,6)$, so dass die direkte Abfrage der Konstrukte mithilfe des "Leitmotivs“ zu keinen deutlichen Verzerrungen geführt haben sollte [3, 14]. Ein weiterer Schwachpunkt ist die 
zeitgleiche Abfrage der Motive und des Trainingsumfangs. Motive stellen aber relativ überdauernde Wertungsdispositionen dar, so dass auch dieser Effekt formal kritisch, aber letztlich sich im Ergebnis nicht relevant ausgewirkt haben dürfte [8]. Methodisch ist die Wahl der Regressionsanalyse zu diskutieren, da die Richtung des Zusammenhangs zwischen Rückenschmerz und Trainingsumfangs nicht eindeutig ist. Beide Richtungen des Zusammenhangs werden in der Literatur diskutiert $[6,19,24]$. Die Wahl der Regressionsanalyse als methodischer Ansatz ist dennoch weitestgehend unkritisch, da Regressionsanalysen keine Informationen über Kausalität und insbesondere die Kausalitätsrichtung liefern.

\section{Fazit für die Praxis}

- Für Frauen ausgerichtete Gesundheitskurse sollten vor allem eines machen: Freude. Auf dem Etikett darf Gesundheit stehen, der Inhalt sollte aber Spaß machen und/oder sich positiv auf die Figur auswirken damit werden gesundheitswirksame Trainingsumfänge eher erreicht als mit dem Motiv Gesundheit.

- Auch für Männer ist die Freude an der sportlichen Aktivität ein wichtiges Motiv. Ein Zusammenhang mit dem Trainingsumfang zeigte sich aber nur mit der Stärke der Rückenschmerzen. Das lösungsorientierte, rückenschmerzreduzierende Training scheint im Vordergrund zu stehen.

- Zukünftige Studien sollten insbesondere den Effekt von Schmerzen als Motivator für Männer überprüfen (bisher wenig beobachtet) und Motivatoren und Barrieren für unterschiedliche Subgruppen ermitteln, damit Anbieter von Präventionsmaßnahmen zielgerichtete Angebote zur Steigerung des Trainingsumfangs machen können.

\section{Korrespondenzadresse}

PD Dr. Gerhard Müller

Geschäftsbereich Vorsorge \& Prävention, AOK Baden-Württemberg

Presselstraße 19, 70191 Stuttgart, Deutschland gerhard.mueller@bw.aok.de

\section{Einhaltung ethischer Richtlinien}

Interessenkonflikt. G. Müller, F. Kunst, N. Stutzig, M. Heinzel-Gutenbrenner, M. Bombana, M. Giurgiu, K. Bös und D. Jekauc geben an, dass kein Interessenkonflikt besteht.

Für diesen Beitrag wurden von den Autoren keine Studien an Menschen oder Tieren durchgeführt. Für die aufgeführten Studien gelten die jeweils dort angegebenen ethischen Richtlinien.

Open Access. Dieser Artikel wird unter der Creative Commons Namensnennung 4.0 International Lizenz veröffentlicht, welche die Nutzung, Vervielfältigung, Bearbeitung, Verbreitung und Wiedergabe in jeglichem Medium und Format erlaubt, sofern Sie den/die ursprünglichen Autor(en) und die Quelle ordnungsgemäßnennen, einen Link zur Creative Commons Lizenz beifügen und angeben, ob Änderungen vorgenommen wurden.

Die in diesem Artikel enthaltenen Bilder und sonstiges Drittmaterial unterliegen ebenfalls der genannten Creative Commons Lizenz, sofern sich aus der Abbildungslegende nichts anderes ergibt. Sofern das betreffende Material nicht unter der genannten Creative Commons Lizenz steht und die betreffende Handlung nicht nach gesetzlichen Vorschriften erlaubt ist, ist für die oben aufgeführten Weiterverwendungen des Materials die Einwilligung des jeweiligen Rechteinhabers einzuholen.

Weitere Details zur Lizenz entnehmen Sie bitte der Lizenzinformation auf http://creativecommons.org/ licenses/by/4.0/deed.de.

\section{Literatur}

1. Alfs C (2014) Sportkonsum in Deutschland: Empirische Analysen zur Allokation von Zeit und Geld für Sport. Springer, Wiesbaden

2. Cohen J (1988) Statistical power analysis for the behavioural sciences, 2. Aufl. Lawrence Erlbaum, Hillsdale

3. Conzelmann A, Lehnert K, Schmid J et al (2012) Das Berner Motiv- und Zielinventar im Freizeitund Gesundheitssport. Anleitung zur Bestimmung von Motivprofilen und motivbasierten Sporttypen. Universität Bern. http://www.zssw.unibe. ch/befragungen/sportberatung/BMZl_klein.pdf. Zugegriffen: 15. Febr. 2020

4. Dahlhaus J (2004) Motivation \& Motivierung zum Alterssport. Niedersächsische Staats- und Universitätsbibliothek, Göttingen

5. European Commission (2010) 334 Sport and physical activity. European Commission study on volunteering. http://ec.europa.eu/public opinion/archives/ebs/ebs_334_en.pdf. Zugegriffen:22. Apr. 2020
6. Ferreira ML, Smeets RJ, Kamper SJ et al (2010) Can we explain heterogeneity among randomized clinical trials of exercise for chronic back pain? A meta-regression analysis of randomized controlled trials. Phys Ther 90(10):1383-1403

7. Finger JD, Mensink GBM, Lange C et al (2017) Gesundheitsfördernde körperliche Aktivität in der Freizeit bei Erwachsenen in Deutschland. J Health Monit 2:37-44

8. GablerH, Nitch JR, Singer R (2004) Einführung in die Sportpsychologie, 4. Aufl. Hofmann, Schorndorf

9. Hurrelmann K, Klotz T, Haisch J (Hrsg) (2008) Lehrbuch Prävention und Gesundheitsförderung. Huber, Berlin, Göttingen, Toronto, Seattle

10. Ingledew DK, Markland D (2008) The role of motives in exercise participation. Psychol Health 23(7):807-828

11. Ingledew DK, Markland D, Ferguson E (2009) Three levels of exercise motivation. Appl Psychol Health Well Being 1(3):336-355

12. International Sport and Culture Association (2015) The economic cost of physical inactivity in Europe. https://inactivity-timebomb.nowwemove.com/download-report/ The $\% 20$ Economic\%20Costs\%20of\%20Physical \%20Inactivity\%20in\%20Europe\%20(June \%202015).pdf.Zugegriffen:22. Apr. 2020

13. von Korff M, Ormel J, Keefe FJ et al (1992) Grading the severity of chronic pain. Pain 50(2):133-149

14. Lehnert K, Sudeck G, Conzelmann A (2011) BMZI - Berner Motiv- und Zielinventar im Freizeit- und Gesundheitssport. Diagnostica 57(3):146-159

15. Lenhard W, Lenhard A (2016) Berechnung von Effektstärken. https://www.psychometrica.de/ effektstaerke.html.Zugegriffen: 15. Febr. 2020

16. da Matta A, Gonçalves FL, Bizarro L (2012) Delay discounting: concepts and measures. Psychol Neurosci 5(2):135-146

17. Müller G, Pfinder M, Clement $M$ et al (2019) Therapeutic and economic effects of multimodal back exercise. J Rehabil Med 51(1):61-70

18. Müller G, Lyssenko L, Giurgiu M et al (2020) How effective and efficient are different exercise patterns in reducing back pain? Eur J Phys Rehabil Med 56(5):585-593

19. Paech J, LippkeS, Ziegelmann JP(2012) Körperliche Aktivität nach der Rehabilitationsbehandlung: die subjektive Beeinträchtigung durch Schmerzen als Barriere oder Motivation. Rehabilitation 51(04):212-220

20. Preuß H, Alfs C, Ahlert G (2012) Sport als Wirtschaftsbranche. Der Sportkonsum privater Haushalte in Deutschland. Springer, Wiesbaden

21. Rhodes RE, Fiala B, Conner M (2009) A review and meta-analysis of affective judgments and physical activity in adult populations. Ann Behav Med 38(3):180-204

22. Ryan RM, Deci EL (2000) Intrinsic and extrinsic motivations: classic definitions and new directions ContempEduc Psychol 25(1):54-67

23. Schüler J, Brunner S, Steiner M (2009) Different effects of activity- and purpose-related incentives on commitment and well-being in the domain of sports. Athl Insight 1(2):1-20

24. Schutzer KA, Graves BS (2004) Barriers and motivations to exercise in older adults. Prev Med 39(5):1056-1061

25. Teixeira PJ, Carraça EV, Markland D et al (2012) Exercise, physical activity, and self-determination theory: a systematic review. Int J Behav Nutr Phys Act 9(78):1-30

26. Vallerand RJ, Rousseau FL (2001) Intrinsic and extrinsic motivation in sport and exercise: a review using the hierarchical model of intrinsic and 


\section{Originalarbeit}

extrinsic motivation. In: Singer RN, Hausenblas HA, Janelle CM (Hrsg) Handbook of sport psychology. Wiley, New York, S389-416

27. Wienke B, Jekauc D (2016) A qualitative analysis of emotional facilitators in exercise. Front Psychol 7:1296

28. Woll A, Bös K (1994) Gesundheit zum Mitmachen: Projektbericht "Gesundheitsförderung in der Gemeinde Bad Schönborn". Hofmann, Schorndorf

29. World Health Organization, Regionalbüro für Europa (2006) Zugewinn an Gesundheit. http:// www.euro.who.int/_data/assets/pdf_file/0009/ 76527/E89306G.pdf.Zugegriffen:14.Jan. 2020

30. World Health Organization, ebrary (2009) Global health risks. World Health Organization, Genf 